\title{
Complicaciones postoperatorias de la serie prospectiva de pacientes con hernioplastia inguinal, en protocolo de hospitalización acortada del Hospital Dr. Gustavo Fricke de Viña del Mar* Complications of inguinal hernia repair
}

\author{
Drs. CEDRIC ADELSDORFER O.1,2, MILENKO SLAKO M.1,2, JOSÉ KLINGER R.1,2, \\ JUAN E. CARTER M.1,2, CARLOS BERGH 0.1,2, EU. CLAUDIA BENAVIDES J.1,2 \\ ${ }^{1}$ Servicio de Cirugía Adultos, Unidad de Cirugía Mayor Ambulatoria. Hospital Dr. Gustavo Fricke de Viña del Mar. \\ ${ }^{2}$ Escuela de Medicina. Universidad de Valparaíso.
}

\section{RESUMEN}

La hernioplastia es la técnica quirúrgica más comúnmente realizada por el cirujano general. Desde la década de los 80, existe un auge por la cirugía ambulatoria en esta patología. Objetivo: Evaluar la incidencia de complicaciones postoperatorias y posibles asociaciones con factores de riesgo, en hernioplastía inguinal electiva en protocolo de hospitalización abreviada. Material y Método: Serie de casos prospectiva de 214 pacientes operados de hernia inguinal, entre abril del 2003 y noviembre del 2005. Se excluyeron los pacientes con ASA III y IV. El análisis estadístico fue realizado mediante la prueba de Chi cuadrado (univariables), y regresión logística (multivariables), SPSS 11.0. p <0,05. Resultados: El promedio de edad fue de 54,3 años (17-84), predominio masculino (90,7\%). Se controló al $100 \%$ de los casos en los primeros 30 días de la intervención, control telefónico $(78 \%)$, y en el policlínico (100\%). Complicaciones postoperatorias presentaron el $14,9 \%$ de los casos, la más frecuente fue el seroma $(4,2 \%)$, la más grave atrofia testicular y dolor crónico invalidante en 1 paciente $(0,47 \%)$, debiendo finalmente realizar orquiectomía. Infección de herida operatoria correspondió al 0,47\%, un paciente ASA I. Los menores de 65 años tuvieron un riesgo relativo de 2,31 de presentar complicaciones. El nivel de satisfacción usuaria fue bueno. El 90\% ha sido controlado hasta agosto del 2006, media de seguimiento de 24 meses y sin presentar recidiva. Conclusiones: La herniorrafia inguinal con hospitalización abreviada, en el hospital Dr. Gustavo Fricke de Viña del Mar, tiene significativamente mayores complicaciones en los menores de 65 años. También ha logrado niveles de satisfacción adecuados, con un mínimo de complicaciones postoperatorias inmediatas.

PALABRAS CLAVE: Hernioplastia inguinal, complicaciones, cirugía ambulatoria.

\section{SUMMARY}

Background: Hernia repair in adults is the most commonly performed surgical procedure by general surgeons. Aim: To evaluate the incidence of postoperative complications in elective inguinal hernioplasty

"Recibido 10 de de Mayo de 2007 y aceptado para publicación el 27 de Julio de 2007.

Correspondencia: Dr. Cedric Adelsdorfer O.

Condell 1176, departamento 70, Valparaíso, Chile

e mail: cedric@adelsdorfer.cl 
with abbreviated hospital stay. Material and Methods: Prospective series of 214 patients aged 16 to 84 years (194 males), operated for inguinal hernia between April 2003 and November 2005. Patients classified as American Society of Anesthesiologists (ASA) III or IV were excluded from the study. Results: All patients were followed during the first 30 days after surgery by telephone and control at the outpatient clinic. Fifteen percent had complications. The most common was seroma in $4 \%$ and the most serious was a testicular atrophy and chronic invalidating pain in one patient, that required an orchidectomy. One patient had a surgical wound infection. Patients of less than 65 years had an odds ratio of 2.13 for complications, when compared with their older counterparts. The level of client satisfaction was good. After 24 months of follow up $90 \%$ of patients were under control and without relapse. Conclusions: Inguinal hernia repair with abbreviated hospital stay has a low incidence of complications.

\section{KEY WORDS: Hernia repair, complications, abbreviated hospital stay.}

\section{INTRODUCCIÓN}

La hernia inguinal es una de las patologías que más frecuentemente requiere de cirugía; se estima entre un $10-15 \%$ de todos las intervenciones quirúrgicas $^{1-3}$. La causa originaria todavía es incierta, se ha establecido una anormalidad en la integridad de la pared abdominal en el área inguinal2,4,5. La hernia inguinal pareciera aparecer en el proceso evolutivo del ser humano, presentando una alta incidencia y por lo tanto es un problema de salud pública con importante implicancias sociales y laborales $^{6}$. Se reconoce que la prevalencia de la hernioplastia es del orden de 280/100.000 habitantes en Estados Unidos, siendo un $70 \%$ de ellas inguinales, y allí existen muchos centros de atención con estadías abreviadas y especialistas en hernias 2,3 . Esta cifra desciende abruptamente en nuestro país, con datos recientemente comunicados en la Revista Chilena de Cirugía, Acevedo y cols., del servicio de salud metropolitano Oriente, quien establece una prevalencia nacional de 136/100.000 habitantes $^{8}$. Dependiendo de la experiencia del cirujano y el tipo de reparación, la tasa de recidiva varía entre el 0,5-10\%. El éxito de la hernioplastia depende, en gran medida, de la comprensión de la anatomía funcional de la pared abdominal y la región inguinal ${ }^{7}$. Pueden enumerarse una gran cantidad de complicaciones, de tipo general, anestésico y las vinculadas con la técnica quirúrgica. El porcentaje de complicaciones mayores es del 5-10\%, muchas de estas son pasajeras, pero algunas pueden presentar un problema socioeconómico. Ha habido una serie de cambios en la cirugía de esta patología, cambios que implican la subespecialización quirúrgica como la forma de poder cumplir con las listas de espera y ofrecer tratamientos con hospitalizaciones abreviadas. En nuestro país las únicas comunicaciones bien tabuladas corresponden a las obtenidas por los grupos de los doctores Acevedo y Arriagada en el servicio de salud metro- politano Oriente, logrando altos niveles de satisfacción usuaria, como reducción de los costos y las listas de espera, por lo que su experiencia es digna de recomendar ${ }^{9-12}$. En nuestro centro esta realidad está institucionalizada hace por lo menos 4 años, cuando se creó la unidad de cirugía ambulatoria mayor (UCAM), que comprende los servicios de cirugía adultos, cirugía infantil, urología y oftalmología. En el caso de cirugía de adultos incluye la patología herniaria, várices y colecistectomías laparoscópicas, las tres con buenos índices de calidad, tema de próximas revisiones. La patología herniaria contempla, a la fecha, aproximadamente 500 procedimientos en 3 años en meses no consecutivos, y nuestra revisión se refiere a ella.

\section{MATERIAL Y MÉTODO}

\section{Diseño del estudio}

Serie de casos prospectiva de todos los pacientes sometidos a hernioplastia inguinal electiva en el período no consecutivo, comprendido entre abril de 2003 y noviembre de 2005.

Población: El total de pacientes beneficiarios del hospital Dr. Gustavo Fricke de Viña del Mar, con diagnóstico de hernia inguinal, sometidos a hernioplastia inguinal electiva del protocolo de hospitalización abreviada, en los meses ya referidos y que cumplían los criterios de inclusión; el total corresponde a 214 pacientes.

Tipo de muestreo: No probabilístico, dado que los pacientes (muestras), se eligieron desde la lista de espera de pacientes para hernioplastia inguinal, con el único condicionante de estar dentro de los criterios de inclusión y el tiempo de espera. Los casos fueron elegidos en forma consecutiva.

Criterios de inclusión: Corresponden a todos los que se eligieron para la cirugía de hospitalización abreviada:

Pacientes mayores 15 años, sin exclusión de edades mayores. 
Corresponder a la clasificación de ASA I y II de la asociación americana de anestesiólogos.

Hernias inguinales unilaterales, las bilaterales fueron operadas en dos tiempos.

Hernias recidivadas unilaterales, las bilaterales fueron operadas en dos tiempos.

Cualquier tipo de hernioplastia.

Cualquier tipo de cirujano, incluidos becarios de la especialidad.

Cualquier tiempo operatorio.

Criterios de exclusión: Todos aquellos pacientes que no cumplieron los criterios de inclusión, es decir, los no candidatos para protocolo de hospitalización abreviada.

Técnica quirúrgica: No se estandarizó la técnica usada, fue resuelta a criterio del cirujano. La más utilizada fue la técnica de Lichtenstein, seguida de Bassini y McVay.

\section{Definición de variables}

Se consignaron los datos de edad, sexo, ASA, hábitos, tipo herniario, tipo de hernioplastia, cirujano, y complicaciones postoperatorias, como también de recidiva y satisfacción usuaria.

Cirugía ambulatoria: todo paciente que no pernocta en el hospital.

Cirugía de hospitalización acortada: pernocta, pero no está más de 24 horas en el hospital.

Clasificación de las hernias inguinales: clasificación tradicional modificada por Zollinger Jr., presentada en la última Clínica Quirúrgica de Norteamérica sobre Hernias 2003. Hace referencia al tipo de defecto, directo o indirecto, y al tamaño de su orificio, pequeño $1,5 \mathrm{~cm}$, mediano $1,5-3 \mathrm{~cm}$, grande mayor $3 \mathrm{~cm}$.

Anestesia: en el 99\% de los pacientes se usó anestesia regional, solo en 2 pacientes anestesia general, por razones técnicas.

Complicación postoperatoria: cualquiera que se presente en los primeros 30 días inmediatos al acto quirúrgico. Se describieron:

Infección herida operatoria: presencia de material purulento, enrojecimiento y fiebre, o cultivo positivo con recuento de colonias mayor o igual a 105 unidades formadoras de colonias (UFC) o la presencia de estreptococo beta lactámico con cualquier recuento.

Seroma: colección de líquido claro sin presencia de gérmenes.

Hematoma: colección hemática.

Neuralgia aguda: aquella que dura menos de 6 semanas.

Neuralgia crónica: aquella que dura más de 6 semanas.
Hidrocele: acumulación de líquido en el área escrotal.

Orquitis isquémica y atrofia testicular: producto de la trombosis del plexo venoso pampiniforme que evoluciona hacia la pérdida del testículo comprometido.

Profilaxis antibiótica: el 100\% de los pacientes recibió algún tipo de profilaxis, 30 minutos antes de iniciar la intervención quirúrgica. Fue realizada con Cefazolina $1 \mathrm{~g}$ endovenoso 0 , en pacientes alérgicos, con Gentamicina $3 \mathrm{mg}$ por kilo endovenoso.

\section{Seguimiento de complicaciones}

El $100 \%$ de los pacientes fue seguido en los primeros 30 días.

Seguimiento telefónico: entre el quinto y séptimo día postoperatorio.

Seguimiento en policlínico de cirugía: entre el décimo quinto y trigésimo día postoperatorio.

\section{Seguimiento a largo plazo}

Encuesta telefónica simple, solo al $90 \%$ de la serie (193 pacientes) en los meses de julio y agosto de 2006. Información acerca de la recidiva herniaria y la satisfacción del usuario mediante una encuesta que considerara definir su grado de satisfacción entre 1-10 puntos, siendo 1 muy malo y 10 excelente.

\section{Análisis estadístico}

El estudio estadístico se realizó mediante el software SPSS versión 11.0 (SPSS Inc., Chicago, Estados Unidos). Se utilizaron para el análisis simple y exploratorio de las variables, las pruebas de promedio, media, desviación estándar, porcentajes.

Para la evaluación de asociaciones entre variables se definieron las pruebas de:

Chi cuadrado $\left(\chi^{2}\right)$ : variables nominales, dicotomizadas, con corrección de Yates, para el análisis univariables.

Regresión logística: con la dicotomización de las variables, para la predicción de la o las asociaciones a partir múltiples variables.

Se consideró un nivel de significancia estadística, a un $\mathrm{p}<0.05$.

\section{RESULTADOS}

La población total corresponde a 214 hernioplastias inguinales, en un período de 27 meses no consecutivos (8-9 meses por año), las características generales de la población se detallan a conti- 
nuación: edad promedio fue de 54,3 años; con un rango entre 16 y 84 años; los mayores de 65 años correspondieron al $30,8 \%$ de la serie. El predominio de los pacientes estudiados fue masculino con un $90,7 \%$. La comorbilidad asociada estuvo presente en el $41,1 \%$ (ASA II), el promedio del IMC fue de 24 , con un $4,7 \%$ de obesos. La caracterización de los pacientes se detalla en la Tabla 1. Se operaron bajo el régimen de cirugía ambulatoria, es decir pacientes fueron dados de alta sin pernoctar en el hospital, el 56,5\% (121 pacientes), mientras que pernoctaron, pero su estancia hospitalaria fue menor de 24 horas, el 43,5\% (93 pacientes), con régimen de hospitalización acortada.

La mayoría de las hernias operadas fueron directas $(58,9 \%)$, las hernias pequeñas (anillos menores a $1,5 \mathrm{~cm}$ ) y medianas (anillos entre 1,5-3 $\mathrm{cm})$, fueron las más frecuentes y sólo se operó un $8,8 \%$ de hernias con anillos mayores a $3 \mathrm{~cm}$, las hernias recidivadas fueron 13 . La técnica mayormente usada fue la reparación con malla, con técnica de Lichtenstein, el tiempo operatorio en promedio fue de 44,4 minutos, con 74,7\% (160 pacientes) de los casos sobre 30 minutos; de estos últimos el $60 \%$ (95), fue operado por un cirujano en formación. Las características del tipo herniario, tamaño, técnica usada se describen con detalle en la Tabla 2. Las distintas técnicas utilizadas se describen en la Tabla 3; importante es considerar que existieron $14 \%$ de hernioplastias con técnicas sin malla, las que fueron realizadas por un cirujano del staff, en pacientes jóvenes, todos bajo los 35 años, sin recidiva hasta la fecha del control telefónico.

El $14,9 \%$ de los pacientes tuvo alguna complicación, la más frecuente fue el seroma de la herida operatoria en 9 casos, seguidas de la neuralgia aguda y la presencia de hidrocele; la más grave fue 1 caso de orquitis isquémica que se siguió por un año con certificación con ecotomografía doppler testicular, y el mismo paciente presentó inguinodinia invalidante, quien debió finalmente terminar en una orquiectomía. La infección de herida operatoria fue en 1 caso, $0,47 \%$, paciente ASA I, sin rechazo

Tabla 1

COMORBILIDAD ASOCIADA

\begin{tabular}{lrc}
\hline & $n=214$ & $\%$ \\
\hline Hipertensión arterial & 49 & 22,9 \\
Diabetes & 7 & 3,3 \\
Tabaquismo & 45 & 21 \\
Sobrepeso (IMC >25 y <30) & 40 & 18,7 \\
Obesidad (IMC >30) & 10 & 4,7 \\
ASA I & 126 & 58,9 \\
ASA II & 88 & 41,1 \\
\end{tabular}

Tabla 2

ANÁLISIS DE FRECUENCIA DE LAS CARACTERÍSTICAS DE LOS PACIENTES OPERADOS, SEGÚN TIPO HERNIARIO, TAMAÑO, TIEMPO DE APARICIÓN, RECIDIVA

\begin{tabular}{lrr}
\hline & $n=214$ & $\%$ \\
\hline Recidivadas & 13 & 6,1 \\
Directas & 126 & 58,9 \\
Indirectas & 88 & 41,1 \\
Pequeña & 84 & 39,3 \\
Mediana & 111 & 51,9 \\
Grande & 19 & 8,8 \\
Espera < 12meses & 144 & 67,3 \\
Espera > 12 meses & 70 & 32,7 \\
\hline
\end{tabular}

del material protésico y buena evolución posterior al drenaje quirúrgico. El detalle de las complicaciones se muestra en la Tabla 4.

El análisis de probables factores de riesgo de presentación de complicaciones postoperatorias a 30 días fue positivo para los menores de 65 años, con un $\mathrm{p}=0,036$ en el análisis univariables y siendo

Tabla 3

TIPO, FRECUENCIA Y CIRUJANO DE LAS HERNIOPLASTÍAS

\begin{tabular}{lrc}
\hline & $n=214$ & $\%$ \\
\hline Lichtenstein & 181 & 84,6 \\
Bassini & 20 & 9,3 \\
Mc Vay & 10 & 4,7 \\
PHS & 3 & 1,4 \\
Uso de malla & 184 & 86 \\
No uso de malla & 30 & 14 \\
Cirujano staff & 98 & 45,8 \\
Cirujano becario & 116 & 54,2 \\
\hline
\end{tabular}

Tabla 4

COMPLICACIONES POSTOPERATORIAS

\begin{tabular}{lcc}
\hline & $n$ & $\%$ \\
\hline Seroma & 9 & 4,2 \\
Hematoma & 6 & 2,8 \\
Hidrocele & 7 & 3,2 \\
Infección herida & 1 & 0,47 \\
Neuralgia aguda (< 6 semanas) & 8 & 3,7 \\
Neuralgia crónica (> 6 semanas) & 1 & 0,47 \\
Orquitis isquémica & 1 & 0,47 \\
Una complicación & 31 & 14,5 \\
Dos o más complicaciones & 1 & 0,47 \\
\hline Total & $32 / 214$ & 14,9 \\
\hline
\end{tabular}


igual de significativo en el análisis multivariables, el Odds Ratio fue 2,63 (1,05<OR <6,75), con un Riesgo Relativo (RR) de 2,31 $(1,09<R R<4,9)$, para los pacientes menores de 65 años. La información de este análisis se evidencia en la Tabla 5.

El control de los pacientes se hizo en forma precoz vía telefónica entre el quinto y séptimo día postoperatorio, consiguiéndose en el $78 \%$ de la serie. El control dentro de los 30 días se efectuó en el $100 \%$ de ellos, de manera presencial, por un becario de cirugía de adultos o médico del staff del servicio de cirugía. Los pacientes que presentaron alguna complicación fueron seguidos por el cirujano tratante hasta el alta de la misma.

El seguimiento a largo plazo no fue presencial y se realizó por vía telefónica con los datos obtenidos desde su ficha de ingreso al estudio y ficha clínica hospitalaria; se logró en el 90\% (193 pacientes), quienes contestaron a la pregunta de recidiva y satisfacción usuaria; el promedio de este seguimiento fue de 24 meses, con una recidiva de $0 \%$ a la fecha de control, la satisfacción usuaria fue buena, con un $90 \%$ (173), sobre los 8 puntos.

\section{DISCUSIÓN}

Las hernias inguinales constituyen uno de los procedimientos más frecuentes practicados en los hospitales generales. Habitualmente los reportes sobre resultados quirúrgicos de la hernia inguinal, son informados en términos de recurrencia de la hernia. Los días de hospitalización, complicaciones tempranas o tardías, dolor postoperatorio y retorno al trabajo, son parámetros que solo ahora se están evaluando, y es así como existe en general poca información acerca de las complicaciones postoperatorias, especialmente referidas en protocolos prospectivos. La incidencia global de estas complicaciones es del orden del $2-15 \%$, según las distintas series: las que tienen menores tasas generalmente corresponden a centros especialistas en hernias ${ }^{13}$, y los que no comunican todas las complicaciones, como por ejemplo la neuralgia aguda (duración menor a 6 semanas) $)^{14,15}$. Nuestros datos resumen las complicaciones en un $14,9 \%$ globalmente y si restáramos los 8 casos de neuralgias agudas, nuestra cifra sería de $11,2 \%$, muy cercana a las mejores series internacionales y nacionales ${ }^{9,16-18}$, en centros docentes. La frecuencia de presentación de las complicaciones postoperatorias sigue el mismo patrón que las presentadas por otros autores $^{14,15,17,19}$. Aunque la mayoría de las series publican que la mayor edad sería un factor de riesgo de eventuales complicaciones postoperatorias, ya existen voces disidentes especialmente desde pro-
Tabla 5

\section{ANÁLISIS UNIVARIABLES Y MULTIVARIABLES, FACTORES DE RIESGO DE APARICIÓN DE COMPLICACIONES POSTOPERATORIAS}

\begin{tabular}{lll}
\hline & $\begin{array}{c}\text { Univa- } \\
\text { riables }\end{array}$ & $\begin{array}{c}\text { Multiva- } \\
\text { riables }\end{array}$ \\
\hline Género & 0,73 & 0,71 \\
ASA & 0,54 & 0,28 \\
Obesidad & 0,8 & 0,93 \\
Edad (< y >de 65 años) & 0,036 & 0,006 \\
Recidiva & 0,9 & 0,97 \\
Tamaño herniario & 0,22 & 0,3 \\
Tiempo aparición (> y <12 meses) & 0,93 & 0,67 \\
Tipo reparación (sin vs con prótesis) & 0,21 & 0,2 \\
Tiempo quirúrgico (> y <30 minutos) & 0,96 & 0,58 \\
Staff vs becados & 0,91 & 0,88 \\
\hline
\end{tabular}

Se consideró significativo $p<0,05$.

tocolos de cirugía ambulatoria ${ }^{20}$, donde no se ha podido demostrar estos preceptos, incluso en nuestra serie resultó ser un factor protector, con un riego relativo aumentado para los menores de 65 años, independiente del ASA, u otros factores de riesgo, inclusive si consideramos el tamaño, el tipo o quién realizó la reparación. El seroma postoperatorio, es una de las complicaciones más frecuentemente reportadas en todas las series, Su incidencia en patología herniaria es del $5 \%{ }^{14,21}$ en hernias primarias y hasta un $10 \%$ en recidivadas; nuestros datos coinciden con estas cifras llegando a un $4,2 \%$ en forma global y a un $7,6 \%$ en recidivadas. La acumulación de líquido en el área escrotal, hidrocele, debiera ser menor del $1 \%$ según datos internacionales ${ }^{14,15,21}$, nosotros encontramos un $3,2 \%$, creemos que, dado que el control postoperatorio fue realizado por una gran cantidad de cirujanos, incluidos cirujanos en formación, muchos de estos supuestos hidroceles realmente correspondían a seromas verdaderos, por lo cual la tasa real de seromas, descontando el $1 \%$ de hidroceles, sería efectivamente de $6,5 \%$. El hematoma postoperatorio es otra complicación frecuente de encontrar, Hidalgo y col21, en su revisión histórica de 1000 hernioplastias demostraron un $2,7 \%$; nosotros en nuestra serie prospectiva, un $2,8 \%$, con 2 casos que se debió intervenir de urgencia, en el postoperatorio inmediato dado el aumento de volumen excesivo y progresivo, ambos casos con buen control posterior. Las neuralgias agudas, menores de 6 semanas no precisan tratamiento especial, no se comunican, ni siquiera en las grandes series de pacientes prospectivos ${ }^{16,17,19}$, y solo encontramos 2 series que describen esta anomalía, la de Büchler ${ }^{18}$, 
con 230 pacientes en hernioplastias efectuadas por cirujanos generales y también en formación, con un $17 \%$, y la de Mayagoitía ${ }^{13}$ referenciada desde la revista Hernia 2006, con 551 pacientes, en un centro especialista, con un 1,45\%. La mayoría de las series que informan sus datos de neuralgias crónicas 0 persistentes están entre el $0-15 \%$, incluso $29 \%$ en las primeras series con cirugía laparoscópica. Nuestra serie presenta 1 caso $(0,47 \%)$, en paciente de 73 años con hernia inguinal directa primaria, que mejoró con el bloqueo anestésico, cifra acorde a los cánones internacionales; solo encontramos el dato de un trabajo prospectivo nacional, con 196 hernioplastias de Lichtenstein, describiendo un 0,9\% de esta complicación ${ }^{9}$. Las infecciones nosocomiales suponen un importante problema de salud que acarrea costos médicos y económicos. Tradicionalmente se considera a la cirugía limpia como un indicador de calidad, control epidemiológico de un servicio de cirugía, incluso es el punto de referencia de la Organización Mundial de la Salud (OMS) y no debe ser mayor al $2 \%$. Sin embargo, es un número alto para la cirugía de la hernia inguinal; la mayoría de las series muestran cifras bajo el $1 \%$, inclusive con $0 \%$, pero pudiendo llegar hasta $9 \% 9,10,18,21,22,23-25$; en nuestra serie correspondió al $0,47 \%$, cifra muy adecuada a los estándares internacionales y nacionales, aunque existen series nacionales que refieren hasta $3 \%$ de infección de herida operatoria ${ }^{9}$. La recidiva es la complicación más importante en la hernioplastia inguinal y se considera adecuado un período de espera de al menos 2 años para que aparezcan la mitad de las recidivas. Nuestra serie muestra un seguimiento de 24 meses con $0 \%$, dato que en el contexto de lo presentado es un número referencial, y por supuesto faltan muchos años para evaluar la real recurrencia. Una comunicación nacional reciente, en seguimiento a 3 años, refiere una tasa de recidiva con técnica de Lichtenstein del orden del $2,7 \%$, también en un hospital docente y con un
$51 \%$ de hernioplastias realizadas por becarios de la especialidad. El resumen de los diferentes estudios se esquematiza en la Tabla 6.

Existe un creciente interés desde la década de los $60^{8}$, pero con mayor fuerza los últimos 20 años, acerca de cirugía con hospitalización abreviada. Es la patología herniaria la más recomendada para este tipo de protocolos, incluso la realizada con anestesia regional, puesto que tiene una recuperación aceptable, como un rango de complicaciones postoperatorias inmediatas muy evaluables por personas neófitas en cirugía ${ }^{8,10-13}$. Nuestra unidad de cirugía ambulatoria recopila pacientes desde oftalmología, urología, cirugía infantil y cirugía de adultos, y de todas las intervenciones realizadas la hernioplastia inguinal ocupa el primer lugar en frecuencia.

No existe a la fecha un consenso que establezca cuando es el mejor momento para realizar una encuesta de satisfacción usuaria; algunos dicen que debería ser en el primer control postoperatorio ${ }^{26}$ dado que rescataría el período de complicaciones precoces, otros establecen un período más largo e incluso con encuestas telefónicas anónimas, para que el paciente tenga una visión más global de su cirugía ${ }^{27}$. En nuestro estudio solo se logró reclutar al $90 \%$, en promedio 26 meses después, logrando en este grupo un $90 \%$ de resultados buenos, lo cual afirma lo comentado por otros autores para este tipo de protocolos $27,53-55$.

\section{CONCLUSIÓN}

La herniorrafia inguinal en un protocolo de hospitalización abreviada, en el Hospital Dr. Gustavo Fricke de Viña del Mar, que tiene mayores complicaciones en los pacientes menores de 65 años en forma significativa.

La tasa de complicaciones es adecuada para un servicio no especialista y centro docente

Tabla 6

COMPARACIÓN DE LAS COMPLICACIONES POSTOPERATORIAS DISTINTAS SERIES

\begin{tabular}{|c|c|c|c|c|c|c|}
\hline & $n$ & $\begin{array}{l}\text { Compli- } \\
\text { caciones }\end{array}$ & $\begin{array}{c}\text { Infección } \\
\text { H operatoria }\end{array}$ & $\begin{array}{l}\text { Neuralgia } \\
\text { aguda }\end{array}$ & $\begin{array}{l}\text { Neuralgia } \\
\text { crónica }\end{array}$ & $\begin{array}{l}\text { Seguimiento } \\
\text { prospectivo }\end{array}$ \\
\hline Hidalgo Cir Esp 2001 & 1000 & $10 \%$ & $1,3 \%$ & No comunica & $2,1 \%$ & No \\
\hline Rodríguez-Cuellar Cir Esp 2005 & 386 & $11,6 \%$ & $1,46 \%$ & No comunica & $2,67 \%$ & $\mathrm{Si}$ \\
\hline Büchler Progress in Surg 1994 & 230 & $14,7 \%$ & 3,04 & $17 \%$ & $0,87 \%$ & No \\
\hline Rutkow, Robbins Surgery 1995 & 2403 & $1 \%$ & $0 \%$ & No comunica & $0 \%$ & No \\
\hline British H Center Surg Clin NA 1998 & 3175 & $5,9 \%$ & $1,3 \%$ & No comunica & $1 \%$ & $\mathrm{Si}$ \\
\hline Mayagoitía Hernia 2006 & 551 & $4,17 \%$ & $0,54 \%$ & $1,45 \%$ & $1,09 \%$ & $\mathrm{Si}$ \\
\hline Benítez Rev Chil Cir 2003 & 196 & $10,7 \%$ & $3,06 \%$ & No comunica & $0,9 \%$ & $\mathrm{Si}$ \\
\hline Acevedo Rev Chil Cir 2005 & 428 & No comunica & $0,2 \%$ & No comunica & No comunica & $\mathrm{Si}$ \\
\hline Viña del Mar 2006 & 214 & $14,9 \%$ & $0,47 \%$ & $3,7 \%$ & $0,47 \%$ & $\mathrm{Si}$ \\
\hline
\end{tabular}


La mortalidad postoperatoria es de $0 \%$. $0,47 \%$

La incidencia de infección de herida es de

Finalmente, el proyecto de cirugía mayor con hospitalización abreviada de las hernias inguinales unilaterales ha demostrado ser posible, con una aceptable tasa de complicaciones, para la población beneficiaria del Hospital Dr. Gustavo Fricke de Viña del Mar.

\section{REFERENCIAS}

1. Lichtenstein IL, Schulman AG, Amid PK. The cause, prevention and treatment of recurrent groin hernia. Surg Clin North Am 1993; 73: 529-544.

2. Rutkow IM. Aspectos epidemiológicos, económicos y sociológicos de la cirugía de la hernia en Estados Unidos en el decenio iniciado en 1990. Surg Clin North Am 1998; 6: 889-900.

3. Rutkow IM. Aspectos demográficos y socioeconómicos de la reparación de las hernias en Estados Unidos en 2003. Surg Clin North Am 2003; 5: 10191028.

4. Bellón JM, Bajo A, Hondurilla NG, Gimeno M, Pascual G. Fibroblasts From the Transversalis Fascia of Young Patients With Direct Inguinal Hernias Show Constitutive MMP-2 Overexpression. Ann Surg 2001; 233: 287-291.

5. Abrahamson J. Causas y fisiopatología de la formación de hernia inguinal primaria y recurrente. Surg Clin North Am 1998; 6: 901-918.

6. Porrero JL, Sánchez-Cabezudo C. Evidencias científicas en el tratamiento de la hernia inguinal. Cir Esp 2002; 72: 157-159.

7. Awad SS, Fagan SP. Current approaches to inguinal hernia repair. J Am Coll Surg 2004; 188: 160-165.

8. Acevedo A, Reyes E, Lombardi J, Robles I. Prevalencia de la cirugía de las hernias inguinales. Rev Chil Cir 2006; 58: 133-137.

9. Benitez S, Hermansen C, Rodríguez E, Andrades $P$, Valenzuela D, Salamanca J. Y cols. Resultados en 196 hernioplastías con técnica de Lichtenstein. Rev Chil Cir 2003; 55: 496-499.

10. Arriagada $E$, Hurtubia $R$, Zenteno $O$, Vargas $C$. Cirugía mayor ambulatoria. Rev Chil Cir 1996; 48: 622625.

11. Arriagada E, Chiang H, Torrejón N, Yañez Z, Torres J, Arriagada L. Cirugía mayor ambulatoria. Rev Chil Cir 2000; 52: 608-612.

12. Acevedo A, Gallego A. Cirugía mayor ambulatoria de las hernias. Experiencia de 5 años en el CRS Cordillera oriente de la ciudad de Santiago. Rev Chil Cir 2004; 56: 166-171.
13. Acevedo A, Gallego A. Cirugía mayor ambulatoria de las hernias. Experiencia de 5 años en el CRS Cordillera oriente de la ciudad de Santiago. Rev Chil Cir 2004; 56: 166-171.

14. Bendavid R. The Shouldice repair. En Schumpelick V, Wantz GE. Inguinal repair. Karger AG, Basel 1995; 222-234.

15. Stephenson BM. Complicaciones de las reparaciones abiertas de las hernias. Surg Clin North Am 2003; 5: 1219-1242.

16. EU Hernia Trialists collaboration. Repair of groin hernia with synthetic mesh. Meta-analysis of randomized controlled trial. Ann surg 2002; 235: 322-332.

17. EU Hernia Trialists collaboration. Mesh compared with non-mesh methods of open groin hernia repair: systematic review of randomized controlled trials. $\mathrm{Br}$ J Surg 2000; 87: 854-859.

18. Büchler MW. Progress in surgery 1994. Editorial Karger

19. Rutkow IM, Robbins AW. Mesh plug hernia repair. A follow up report. Surgery 1995; 117: 597-608.

20. Ciga MA, Oteiza F, Ortiz H. Estudio prospectivo de las complicaciones de la cirugía de la hernia en función de la edad de los pacientes. Cir Esp 2004; 75 : 204-206.

21. Hidalgo M, Castellón C, Figueroa J M, Eymar J L, Moreno González E. Complicaciones de la cirugía de las hernias. Cir Esp 2001; 69: 217-223.

22. Gil P, Esteban E, Legido P, Gago P, Pastor E. Tasa de infección en cirugía limpia: seguimiento hasta 30 días tras la intervención. Cir Esp 2005; 77: 226-229.

23. Rutkow IM, Bobbins AW. Tension-free inguinal herniorraphy: A preliminary report on the mesh plug technique. Surgery 1993; 114: 123.

24. Taylor E, Byrne DD, Leaper D, Karran S, Brownw MK, Mitchell KJ, et al. Antibiotic prophylaxis an open groin hernia repair. World J Surg 1997; 21: 811-815.

25. Granell J. Prótesis de pared abdominal e infección quirúrgica. Cir Esp 1998; 63: 333-335.

26. Hawn M, Itani K, Giobbie-Hurder A, McCarthy M, Jonasson O, Neumayer L. Patient-reported outcomes after inguinal herniorraphy. Surgery 2006; 140: 198-205.

27. Jiménez A, Elia M, Gracia J, Artigas C, Lamata F, Martínez M. Indicadores de calidad asistencial en cirugía mayor ambulatoria. Cir Esp 2004; 76: 325330.

28. Duce AM, Arrieta F, Tobaruela E, Villeta R, Mugüerza J, Noguerales F. When to return to work alter groin hernia repair. Ambulatory Surg 2000; 8: 89-91.

29. Lau $H$, Lee $F$. Determinant factors of pain after ambulatory inguinal herniorraphy: a multi-variate analysis. Hernia 2001; 5: 17-20. 